\title{
SirT1 modulates the estrogen-insulin-like growth factor-1 signaling for postnatal development of mammary gland in mice
}

\author{
Hongzhe Li $i^{1,2}$, Grace K Rajendran ${ }^{1,2}$, Ninning Liu ${ }^{1,2}$, Carol Ware ${ }^{3}$, Brian P Rubin ${ }^{4,5}$ and \\ Yansong $\mathrm{Gu}^{1,2}$
}

\author{
1Department of Radiation Oncology, University of Washington School of Medicine, Seattle, WA 98195, USA \\ 2Department of Immunology, University of Washington School of Medicine, Seattle, WA 98195, USA \\ 3Department of Comparative Medicine, University of Washington School of Medicine, Seattle, WA 98195, USA \\ ${ }^{4}$ Department of Pathology, University of Washington School of Medicine, Seattle, WA 98195, USA \\ ${ }^{5}$ Department of Molecular Genetics, Lerner Research Institute, Cleveland, OH 44195, USA \\ Corresponding author: Yansong Gu, ygu@u.washington.edu
}

Received: 21 Aug 2006 Revisions requested: 6 Oct 2006 Revisions received: 9 Nov 2006 Accepted: 3 Jan 2007 Published: 3 Jan 2007

Breast Cancer Research 2007, 9:R1 (doi:10.1186/bcr1632)

This article is online at: http://breast-cancer-research.com/content/9/1/R1

(c) 2007 Gu et al.; licensee BioMed Central Ltd.

This is an open access article distributed under the terms of the Creative Commons Attribution License (http://creativecommons.org/licenses/by/2.0), which permits unrestricted use, distribution, and reproduction in any medium, provided the original work is properly cited.

\begin{abstract}
Introduction Estrogen and insulin-like growth factor-1 (IGF-1) play important roles in mammary gland development and breast cancer. SirT1 is a highly conserved protein deacetylase that can regulate the insulin/IGF-1 signaling in lower organisms, as well as a growing number of transcription factors, including NF- $\mathrm{B}$, in mammalian cells. Whether SirT1 regulates the IGF-1 signaling for mammary gland development and function, however, is not clear. In the present study, this role of SirT1 was examined by studying SirT1-deficient mice.
\end{abstract}

Methods SirT1-deficient (SirT1ko/ko) mice were generated by crossing a new strain of mice harboring a conditional targeted mutation in the SirT1 gene (SirT1 ${ }^{\mathrm{co} / \mathrm{co}}$ ) with CMV-Cre transgenic mice. Whole mount and histology analyses, immunofluorescence staining, immunohistochemistry, and western blotting were used to characterize mammary gland development in virgin and pregnant mice. The effect of exogenous estrogen was also examined by subcutaneous implantation of a slow-releasing pellet in the subscapular region.

Results Both male and female SirT1ko/ko mice can be fertile despite the growth retardation phenotype. Virgin SirT1 ${ }^{\text {ko/ko mice }}$ displayed impeded ductal morphogenesis, whereas pregnant SirT1ko/ko mice manifested lactation failure due to an underdeveloped lobuloalveolar network. Estrogen implantation was sufficient to rescue ductal morphogenesis. Exogenous estrogen reversed the increased basal level of IGF-1 binding protein-1 expression in SirT1 $1{ }^{\mathrm{k} / \mathrm{ko}}$ mammary tissues, but not that of $I \kappa B \alpha$ expression, suggesting that increased levels of estrogen enhanced the production of local IGF-1 and rescued ductal morphogenesis. Additionally, TNF $\alpha$ treatment enhanced the level of the newly synthesized $\mathrm{I} \mathrm{B} \mathrm{B} \alpha$ in SirT1ko/ko cells. SirT1 deficiency therefore affects the cellular response to multiple extrinsic signals.

Conclusion SirT1 modulates the IGF-1 signaling critical for both growth regulation and mammary gland development in mice. SirT1 deficiency deregulates the expression of IGF-1 binding protein-1 and attenuates the effect of IGF-1 signals, including estrogen-stimulated local IGF-1 signaling for the onset of ductal morphogenesis. These findings suggest that the enzymatic activity of SirT1 may influence both normal growth and malignant growth of mammary epithelial cells.

\section{Introduction}

Mammalian SirT1 belongs to a family of nicotinamide adenine dinucleotide-dependent histone deacetylases $[1,2]$. SirT1 is most closely related to yeast Sir2, the founding member of the evolutionarily conserved Sir2 family. Yeast Sir2 is required for silencing transcription at the telomeric region and mating type loci, and for suppression of ribosomal DNA recombination $[3,4]$. The expression of an extra copy of Sir2 in either yeast

$\overline{\mathrm{Atm}}=$ ataxia telangiectasia; BrdU = bromodeoxyurdine; CMV = cytomegalovirus; ER $\alpha=$ estrogen receptor alpha; FoxO = forkhead box 'other' protein; $\mathrm{GH}=$ growth hormone; IGF-1 = insulin-like growth factor-1; IGFBP-1 = insulin-like growth factor-1 binding protein-1; $\mathrm{H}$ \& $\mathrm{E}=$ hemotoxylin and eosin; IIS = insulin/insulin-like growth factor-1 signaling; I $\mathrm{KB} \alpha=$ inhibitors of NF- $\kappa B$ alpha subunit; IKK $\alpha=I \kappa B$ kinase alpha; MEF = murine embryonic fibroblast; NF= nuclear factor; PCR = polymerase chain reaction; RANK = receptor activator of NF- $\kappa B$; Sir $=$ silencing information regulator; TEB = terminal end bud; TNF = tumor necrosis factor. 
mother cells or multicell organisms such as nematodes can significantly extend the lifespan $[5,6]$. Inactivation of Sir2 enhances stress resistance and extends chronological lifespan of nondividing yeast cells, which is opposite to the requirement for Sir2 function in the reproductive lifespan [7]. Whether SirT1 regulates the reproductive lifespan and/or the chronological lifespan in mammals remains unknown.

Sir2 is an integral part of an evolutionarily conserved insulin/ insulin-like growth factor-1 (IGF-1) signaling (IIS) system in worms (Caenorhabditis elegans), fruit flies (Drosophila), mice, and humans $[8,9]$. The IIS system includes membrane-bound receptors, cytoplasmic kinases, and nuclear transcription factors. To maintain the proper expression of the effector genes for the IIS system, these conserved components form a sophisticated regulatory system, which centers on a family of forkhead transcription factors (forkhead box 'other' proteins (FoxOs)), and operates on two levels. On one level, SirT1mediated protein deacetylation attenuates the transcriptional activity of nuclear FoxO transcription factors [10-12]. On the second level, the FoxO transcription factors can be sequestered within the cytoplasm when phosphorylated by activated Akt kinases in response to insulin and IGF-1 signals [13]. Conceivably, the IIS system senses the levels of insulin and IGF-1 and negatively regulates the expression of the effector genes. The IIS system is responsible for food storage, stress tolerance, and longevity in lower organisms, such as C. elegans $[8,9,14]$. In more advanced species, steroid hormones evolved to regulate the IIS system [15]. In mice and humans, the IGF1 signaling of the IIS system mediates local effects for growth and hormonal regulation for multiple tissues, including mammary glands $[16,17]$.

Mammalian SirT1 has evolved to modify the activity of a growing number of transcription factors, including $\mathrm{p} 53, \mathrm{NF}-\mathrm{KB}$, and PGC-1 $\alpha$, suggesting that SirT1 functions in a wide range of cellular responses to stress, inflammation, and nutrients [1821]. SirT1-deficient mice display characteristic phenotypes of perinatal death and growth retardation as well as other diverse phenotypes, such as eye defects, with varying severity $[22,23]$. The underlying causal mechanism for these phenotypes, however, remains unknown. We recently generated SirT1-deficient (SirT1 ko/ko) mice and found that both male and female SirT1 ko/ko mice can be fertile, which is in contrast to the sterile phenotypes observed in one strain of SirT1-deficient mice [22]. This led to our study of the link between SirT1 and IGF-1 signaling using the mammary gland as a model organ.

The mammary gland is a unique organ because it develops after birth and undergoes dynamic changes throughout the reproductive lifespan of a female. At the onset of puberty, ovarian estrogen stimulates ductal morphogenesis during which mammary epithelial progenitor cells differentiate and proliferate while interacting with adipocytes and stromal cells within mammary fat pad $[17,24,25]$. Ovarian estrogen, in synergy with pituitary growth hormone $(\mathrm{GH})$, stimulates stromal cells to produce local IGF-1. The local IGF-1, but not liver-produced systemic IGF-1, provides a paracrine signal for commencing ductal morphogenesis [26]. Mice lacking GH, estrogen, IGF$1, \mathrm{GH}$ receptor, or estrogen receptor alpha $(E R \alpha)$ fail to undergo postnatal ductal morphogenesis [17,24-31], indicating that both steroid hormones and IGF- 1 are on the common pathway for a critical developmental checkpoint. Once the arborated ductal network is established, cycles of differentiation, proliferation, and death of secretory alveolar epithelium repeat with each pregnancy $[17,24,25]$.

In the present article we report the finding and characterization of impeded ductal morphogenesis in virgin SirT1 ko/ko mice and lactation failure in SirT1 ko/ko mothers. The characterization of these phenotypes has identified a SirT1-dependent regulatory mechanism by which SirT1 modulates the effectiveness of the estrogen-IGF-1 signaling for mammary gland development. The estrogen-IGF-1 signaling is defined as the ovarian estrogen-regulated, stromal cell-produced local IGF-1 signal for stimulating mammary epithelial cells.

\section{Materials and methods Mice}

A previously described SirT1 targeting construct, KOII [23], was used to generate mice harboring a conditional targeted mutation in the SirT1 gene (SirT1 ${ }^{\text {co/co }}$ mice) (see Additional file 1). The breeding of SirT1 ${ }^{\mathrm{co} / \mathrm{co}}$ mice and CMV-Cre transgenic mice results in mice harboring a germline-transmitted deletion of exon 4 of the SirT1 gene (SirT1+/ko mice). Both SirT1 co/co mice and SirT1+/co mice were used to establish breeding colonies for generating SirT1 1 co/co and SirT1 ko/ko mice, respectively. Both SirT1 1 co/co mice and SirT1 ko/ko mice were in a mixed 129SvJ/C57B6 background. Mice were housed in a specialpathogen-free facility and all procedures were approved by the University of Washington Animal Care and Use Committee. A PCR-based genotyping method was established to identify the wild-type, co, and ko loci of the SirT1 gene using three primers: 5' co primer, 5'-GGTTGACTTAGGTCTTGTCTG; 5' ko primer, 5'-AGGCGGATTTCTGAGTTCGA; 3' primer, 5'CGTCCCTTGTAATGTTTCCC. Murine embryonic fibroblasts (MEFs) were isolated from the embryos between embryonic day 12.5 and embryonic day 14.5. The body weight was measured once a week.

\section{Serum insulin-like growth factor-1}

A commercial radioimmunoassay (ALPCO Inc., Windham, $\mathrm{NH}$, USA) was used. Samples $(300 \mu l)$ were prepared via an alcohol extraction followed by a 2-day disequilibrium incubation at $4^{\circ} \mathrm{C}$. Samples were measured in duplicate and data were calculated as the samples were counted. Six standards ranging from 10 to $500 \mathrm{ng} / \mathrm{ml}$ were used to generate the standard curve. The lower limit of detection was $10 \mathrm{ng}$. 


\section{Survival assay}

To determine the sensitivity of embryonic stem cells after ionizing radiation or hydrogen peroxide treatment, 300 embryonic stem cells were seeded onto a $10-\mathrm{cm}$ plate for 24 hours. The plates were either exposed to a ${ }^{137} \mathrm{Cs}$ source at indicated doses or were treated with embryonic stem media containing hydrogen peroxide at indicated concentrations for 30 minutes. Seven days after irradiation or hydrogen peroxide treatment, the embryonic stem colonies stained with crystal violet were counted.

\section{Estrogen implantation}

Estrogen pellets in the form of $17 \beta$-estradiol, at $0.1 \mathrm{mg}$, and the 21-day releasing time were obtained from Innovative Research (Sarasota, FL, USA). One pelletwas subcutaneously implanted in the subscapular region ofindividual female mice using a sterilizedmetal trochar (Innovative Research).

\section{Whole mount analysis}

The inguinoabdominal mammary fat pads were spread on microscope slides, fixed in Carnoy's fixative overnight, hydrated and stained with carmine alum stain (Sigma, St. Louis, MO, USA) overnight, and were then dehydrated, treated with xylene to remove fat, and mounted with Secure Mount (Fisher Scientific, Pittsburgh, PA, USA) and cover slips.

\section{Histological, immunohistochemical, and immunofluorescence analyses}

The mice were given a single dose of bromodeoxyurdine (BrdU) at $100 \mathrm{~g} / \mathrm{kg}$ body weight via intraperitoneal injection 2 hours before euthanasia. The mammary fat pads, as well as other tissues, were collected and fixed in $10 \%$ formalin solution (Fisher Scientific). Paraffin-embedded sections were prepared at $4 \mu \mathrm{m}$ thickness followed by standard $\mathrm{H}$ \& $\mathrm{E}$ staining for histological analysis and by immunohistochemical staining for BrdU-labeled cells (Sigma) and apoptotic cells (Promega, Madison, WI, USA). To detect the presence of mouse milk proteins, a rabbit anti-mouse milk specific protein antibody (Nordic Immunology, Tillburg, The Netherlands) was used for immunofluorescent staining, followed by Texas Red anti-rabbit secondary antibody (Molecular Probes) and DAPI staining for nuclei (Molecular Probe, Eugene, OR, USA). Microscopic analyses of all histological findings were carried out on an AxioVert 200M microscope with AxioVision 4.5 software (Carl Zeiss, München-Hallbergmoos, Germany).

\section{Protein analysis}

Protein extracts were prepared from mammary tissues as well as from MEFs. The following primary antibodies were used: anti-SirT1 (Upstate Biotechology, Lake Placid, NY, USA), antiIGF-1 binding protein-1 (IGFBP-1) (R\&D Systems, Minneapolis, MN, USA), and anti-actin and anti-IKB $\alpha$ (Santa Cruz Biotechnology, Santa Cruz, CA, USA). The corresponding secondary antibodies were horseradish peroxidase-conju- gated anti-rabbit (Pierce, Rockford, IL, USA), anti-rat, and antigoat (Santa Cruz Biotechnology).

\section{Results \\ Growth retardation in SirT1ko/ko mice}

The conditional targeted SirT1 mutant mice (SirT1 co/co mice) carry an insertion mutation of the neomycin-resistant gene and lox sequences in the SirT1 gene flanking exon 4 that encodes a conserved Sir2 motif (Figure 1a). The mutation does not

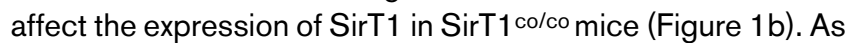
expected, SirT1 co/co mice are phenotypically indistinguishable from wild-type mice. To convert the SirT1 co allele into the SirT1 ko allele, SirT1 1 co/co mice were crossed with CMV-Cre transgenic mice to generate SirT1 heterozygotes carrying the SirT1+/ko, CMV-Cre ${ }^{+}$genotype. The expression of the CMVCre transgene catalyzes the deletion of exon 4 in most lineages of cells, including germ cells (Figure 1a). This SirT1 ko allele should be identical to the previously described $\Delta$ ex4 mutation [23]. SirT1+/ko, $\mathrm{Cre}^{+}$mice were backcrossed with wild-type mice to generate the mice harboring a germlinetransmitted deletion mutation (that is, SirT1+/ko mice). The breeding of SirT1+/ko male mice and SirT1+/ko female mice resulted in SirT1ko/ko mice. The cells derived from either SirT1 ${ }^{+/ k o}$ mice or SirT1 ko/ko mice expressed a SirT1 mutant protein due to the inframe deletion of exon 4 (Figure 1b). This SirT1 mutant protein may not be functional, however, since SirT1+/ko mice are phenotypically indistinguishable from wildtype mice and $\operatorname{SirT} 1 \Delta^{\mathrm{ex} 4 / \Delta \mathrm{ex} 4}$ mice were phenotypically identical to SirT1 null mice [23].

Similar to other strains of SirT1-deficient mice, nearly twothirds of SirT1ko/ko newborns die shortly after birth and the majority of surviving SirT1 ko/ko mice manifest growth retardation (Table 1 and Figure 1c) [22,23]. Growth retardation may result from a systemic defect in hormonal regulation, DNA double-strand break repair, or other causal mechanisms. We found that SirT1 ko/ko mice have reduced levels of serum IGF-1 (Figure 1d). IGF-1 often acts as a local effector for pituitary $\mathrm{GH}$. The serum level of $\mathrm{GH}$ in SirT1ko/ko mice, however, appeared to be within the normal range (data not shown). On the other hand, yeast Sir2 and its associated Sir complex, as well as mouse SirT6, have also been implicated in DNA double-strand break repair and the maintenance of chromosome stability [32-34]. We found that SirT1ko/ko embryonic stem cells do not have increased sensitivity to either ionizing radiation or hydrogen peroxide treatment when compared with wild-type cells, or with positive control cells such as Ku70deficient or Atm-deficient cells (Figure 1e,f). Both Ku70-deficient mice and Atm-deficient mice display growth retardation phenotypes $[35,36]$. The growth retardation phenotype in SirT1ko/ko mice is therefore probably not due to a defect in DNA damage repair, but rather results from a deficit in IGF-1 signaling. 
Figure 1

(a)

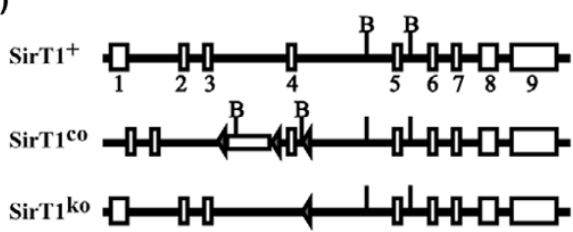

(b)

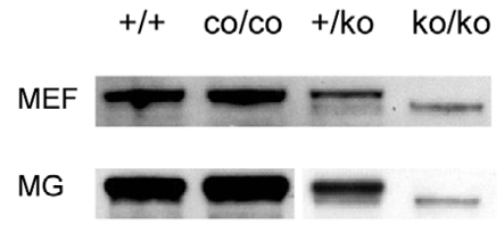

(c)

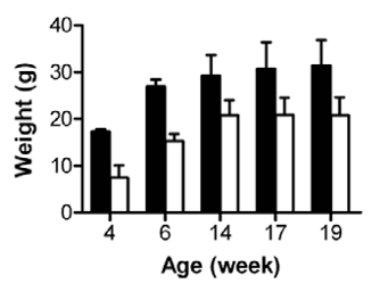

(d)

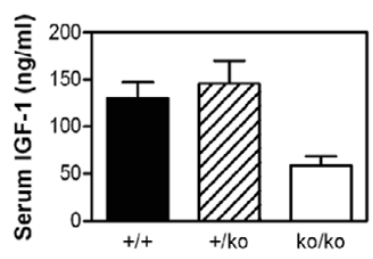

(e)

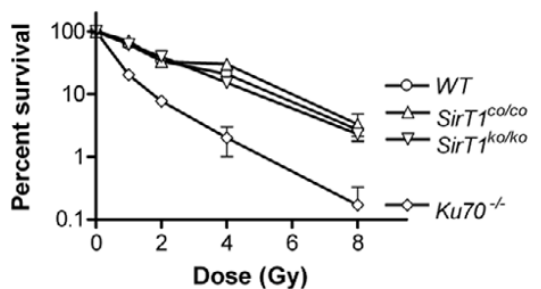

(f)

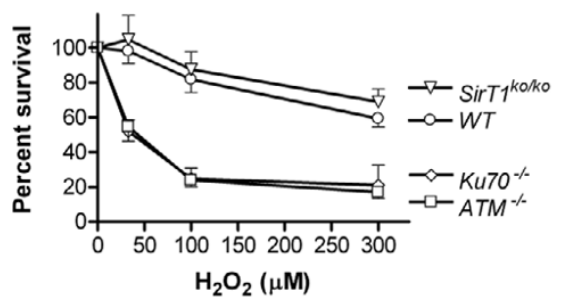

Generation and characterization of SirT1 ${ }^{\mathrm{co} / \mathrm{co}}$ and SirT1 ko/ko mice. (a) Mouse SirT1 wild-type allele (SirT1+), conditional targeted allele (SirT1 co), and knockout allele (SirT1 ko). B, BamHl. (b) Western blot analysis of SirT1 expression in wild-type (+/+), conditional targeted (co/co), heterozygote (+/ko), and knockout (ko/ko) murine embryonic fibroblasts (MEFs) and mammary tissues (MG). (c) Growth retardation in surviving SirT1 ko/ko mice (open bar) using sibling mice or age-matched mice of control genotypes (filled bar). (d) The serum levels of insulin-like growth factor-1 (IGF-1) in wild-type (+/+), SirT1+/ko (+/ko), and SirT1ko/ko (ko/ko) mice. (e) The survival curves of wild-type (WT), SirT1 co/co, SirT1 ko/ko, and Ku70\% embryonic stem cells after ionizing radiation. (f) The survival curve of wild-type (WT), SirT1 ko/ko, Ku70 ${ }^{-/}$, and Atm ${ }^{-/}$embryonic stem cells after treatment with hydrogen peroxide.

\section{Lactation failure}

Both male and female SirT1 ko/ko mice can be fertile, which is in contrast with the reported sterile phenotype in one strain of SirT1-deficient mice [22]. When male SirT1ko/ko mice impregnated female SirT1+/ko mice, the number of surviving SirT1 ko/ko offspring was reduced due to the partial perinatal lethal phenotype (Table 1). In a reciprocal approach, male SirT1+/ko mice can also impregnate female SirT1ko/ko mice (Table 1). After parturition, SirT1 ko/ko mothers exhibited normal nursing behavior. The pups did not survive for more than 3 days after birth, however, unless they were immediately removed and put under the care of a foster mother. All pups died of dehydration or starvation as a result of lack of milk in their stomachs.

To determine whether the SirT1 ko/ko mothers encountered a lactation defect, whole mount and histological analyses were used to characterize the morphological changes in the mam- mary glands. We found that virgin SirT1 ko/ko mice displayed impeded ductal morphogenesis up to 9 months of age, while age-matched virgin wild-type mice displayed extensive ductal elongation and branching (Figure 2a). The absence of ductal morphogenesis persisted in pregnant SirT1 ko/ko mice up to day 13 of pregnancy (Figure 2b). Despite the fact that pregnancy can induce ductal morphogenesis at the late stage of pregnancy, underdeveloped mammary glands in SirT1 ko/ko female mice cause a severe deficit in milk production, as shown by histological analysis as well as the immunofluorescence analysis using an anti-milk antibody (Figure 2b,c).

\section{Pregnancy-induced mammary gland development}

To characterize the developmental defect leading to lactation failure, we analyzed the mammary glands of SirT1 ko/ko female mice on lactation day 1. Terminal end buds (TEBs) are clubshaped transitional structures (see Figure $3 a$, upper panel). In

Table 1

Perinatal lethality, fertility, and lactation defect in SirT1ko/ko mice

\begin{tabular}{lllllll}
\hline Parents & & Pups & & & \\
\hline Male & Female & Litter & Survivors & $+/+$ & ko & \\
\hline$+/$ ko & $+/$ ko & 47 & 340 & 101 & 208 & 31 \\
ko/ko $(n=3)$ & $+/$ ko & 11 & 55 & - & 45 & 10 \\
$+/$ ko & ko/ko $(n=5)$ & 8 & 0 & - & (13) & (13) \\
\hline
\end{tabular}

More than 10 pairs of SirT1+/ko male mice and SirT1 $1^{+/ k o}$ female mice were used for breeding. Data in parentheses indicate the number of pups of this genotype found dead within 3 days after their birth. aTotal number of surviving pups at weaning (postnatal day 21). 
Figure 2

(a)
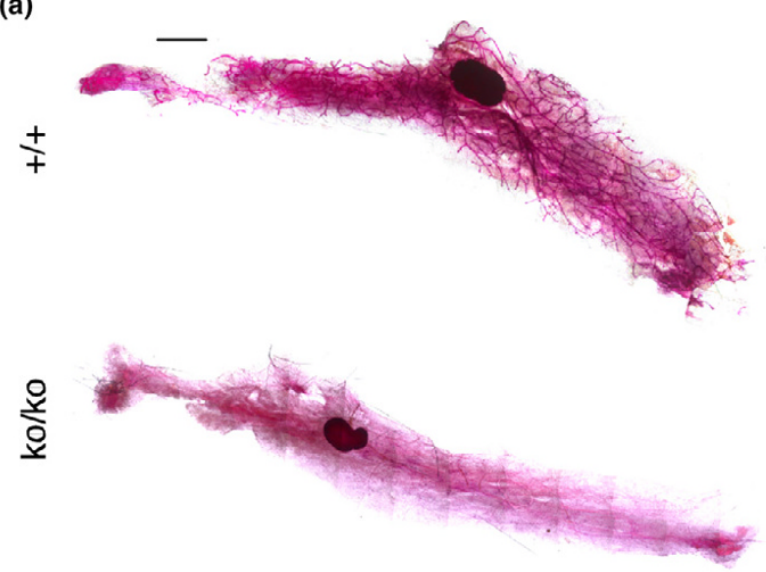

(b)

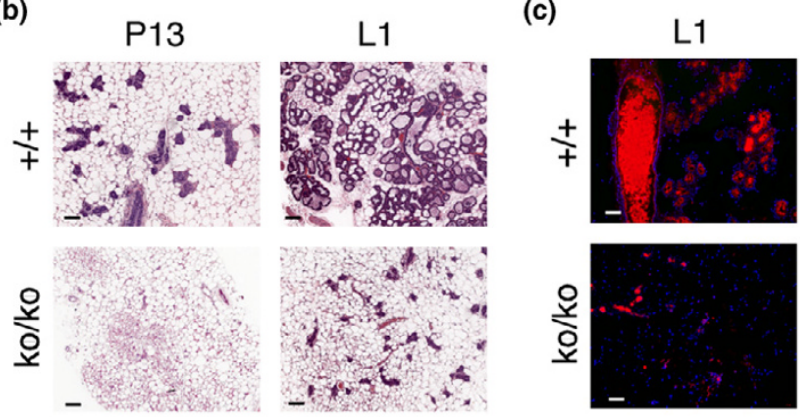

Lactation failure in SirT1 ko/ko mice. (a) Whole mount analysis of the mammary tissues from virgin wild-type $(+/+)$ and SirT1 ko/ko mice $(\mathrm{ko} /$ ko). Scale bar $=2 \mathrm{~mm}$. (b) Histological analysis of the mammary tissues harvested from wild-type $(+/+)$ and SirT 1 ko/ko female mice on either day 13 of pregnancy (P13) or lactation day 1 (L1). Scale bars $=$ $100 \mu \mathrm{m}$. (c) Immunofluorescence staining of milk production in the mammary tissues from wild-type (+/+) and SirT1 ko/ko mice on L1. Red and smear stains, mouse milk proteins; blue dots, nuclear staining of adipocytes, epithelial cells, and other cells in mammary tissues. Scale bars $=100 \mu \mathrm{m}$.

wild-type female mice, TEBs form and precede ductal elongation and branching at the onset of puberty $[17,24,25]$. When TEBs/ducts reach the edge of mammary fat pads, TEBs regress as shown in the virgin mice in Figure 3a (upper panel). Interestingly, the transitional TEBs can be readily identified in SirT1 ko/ko female mice on lactation day 1 , manifesting as either newly formed TEBs or as TEBs attached to developing ductal and alveolar structures (Figure $3 \mathrm{a}$, lower panel). Moreover, SirT1 ko/ko mice displayed varying degrees of ductal development, ranging from a lack of any ductal structure to a fully developed ductal network, the latter of which is comparable with the morphology and cellularity of wild-type mammary tissues on day 13 of pregnancy (Figure 3a). These observations indicated that pregnancy could rescue impeded ductal morphogenesis in virgin SirT1 ko/ko mice.

Normal alveolar morphogenesis takes place under the control of additional hormones and growth factors during pregnancy and lactation [17,24,25]. These coordinated efforts are necessary to support a densely saturated lobuloalveolar system for secreting milk after parturition (see wild-type mice on lactation day 1 in Figures $2 b$ and 3a). In some SirT1 ko/ko mice, the alveolar morphogenesis was initiated, as indicated by the presence of a few secretory alveolar epithelial cells within scattered alveolar structures sprouted from a well-established ductal branching network (Figures $2 \mathrm{c}$ and $3 \mathrm{a}$, lower panel). The extent of alveolar morphogenesis in SirT1 ko/ko mice was inadequate, however, as shown by the severe deficit in milk production when compared with that in wild-type mice (Figure 2c).

\section{Exogenous estrogen-induced ductal morphogenesis}

To test whether the increased levels of estrogen in pregnant mice were sufficient to induce ductal morphogenesis in SirT1 ko/ko mice, a single estrogen pellet was implanted in the subscapular region of each virgin SirT1ko/ko mouse and the mammary tissues were analyzed 14 and 21 days after the implantation. By day 14, wild-type mice displayed ductal side branching reminiscent of the morphological changes during early pregnancy (Figure 4a, left panel). In contrast, SirT1ko/ko mice showed TEBs and ductal elongation, which are the characteristic features of ductal morphogenesis in pubertal wildtype mice. By day 21, ductal elongation and side branching were restored in SirT1ko/ko mice and the extent of ductal morphogenesis was indistinguishable between wild-type and SirT1 ko/ko female mice (Figure $4 \mathrm{a}$, right panel). These observations clearly indicated that exogenous estrogen alone is sufficient to rescue ductal morphogenesis in virgin SirT1 ko/ko mice. Furthermore, the presence of transitional TEBs on day 14 and of ductal side branching on day 21 suggested that the characteristic features of mammary gland development at puberty and during early pregnancy could be coupled in response to increasing levels of estrogen. Increased levels of estrogen, either during pregnancy or through implantation, were therefore sufficient to stimulate the differentiation of epithelial progenitor cells and ductal morphogenesis in virgin SirT1ko/ko mice.

\section{Concurrent mammary epithelial cell proliferation and differentiation}

The finding of pregnancy-induced mammary gland development prompted us to investigate how mammary epithelial pro-

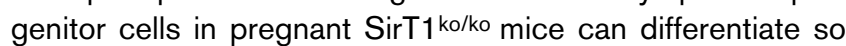
rapidly. Both BrdU labeling and the TUNEL (Terminal deoxynucleotidyl transferase mediated dUTP Nick End Labeling) assay were used to measure the cell proliferation and apoptosis in the mammary tissues, respectively. We found in SirT1ko/ko mammary tissues that about one-third of the cells in TEBs were BrdU-positive as compared with the number of BrdUpositive cells in the newly differentiated ductal or alveolar epithelial cells (Figure $5 \mathrm{a}$ (upper panel), 5b). This BrdU-positive finding differs from that in wild-type mammary tissues, in which less than $3 \%$ of ductal epithelial cells and about $25 \%$ of alve- 


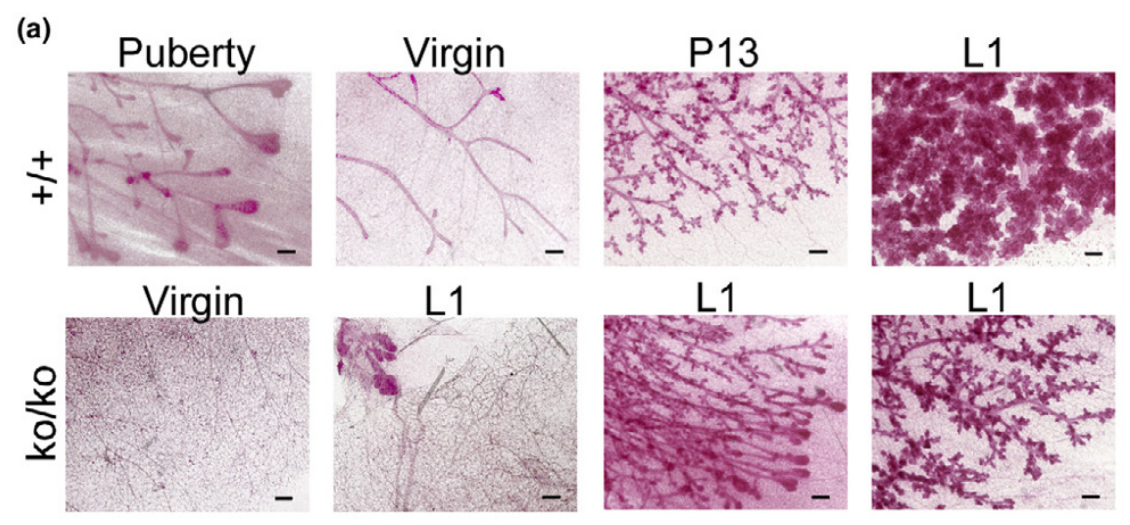

(b)

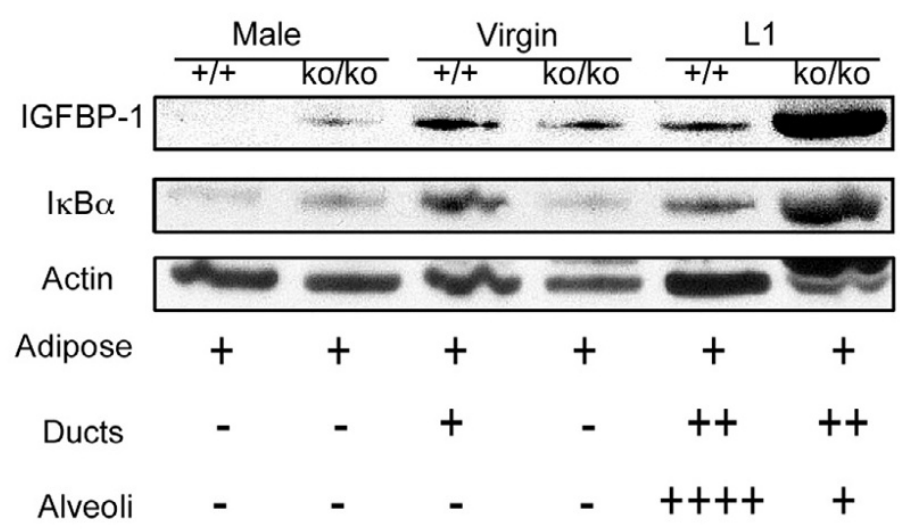

Pregnancy-induced ductal morphogenesis. (a) Whole mount analysis of mammary gland development. Upper panel: wild-type (+/+) mice show terminal end buds (TEBs) at the onset of puberty, elongated ducts (virgin mice), site branching during pregnancy (P13), and lobuloalveolar structures for milk production on lactation day 1 (L1). Lower panel: virgin SirT1 ko/ko (ko/ko) mice show impeded ductal morphogenesis. Pregnancyinduced ductal morphogenesis manifests transitional TEBs, ductal elongation, and side branching with variety in three SirT1 ko/ko mice on L1. All scale bars $=200 \mu \mathrm{m}$. (b) Western blot analysis of both insulin-like growth factor-1 binding protein-1 (IGFBP-1) and IKB $\alpha$ in mammary tissues of male mice, virgin mice, and L1 female mice of wild type $(+/+)$ and SirT1 ko/ko $(\mathrm{ko} / \mathrm{ko})$. Actin is used as a loading control. Lower panel scores the estimated density of indicated lineages of cells in the protein extracts, which are based on the morphological analyses in (a).

olar epithelial cells were BrdU-positive. The newly generated ductal epithelial cells in SirT1ko/ko mice formed elongated ducts and side branches, while pushing TEBs towards the edge of mammary fat pad (Figure 3a, lower panel). The newly generated ductal epithelial cells can consequently further differentiate into a few observed alveolar cells in which milk proteins were detected (Figure 2c). These observations suggested that the proliferation and differentiation of epithelial progenitor cells, which is programmed to take place at different times of a female's reproductive life, could be concurrent at a late stage of pregnancy in SirT1 ko/ko mice.

Apoptosis is a part of the self-renewal and remodeling processes in normal mammary tissues [17,24,25]. Apoptotic epithelial cells can be readily identified in both ductal and alveolar epithelium of SirT1 ko/ko mice on lactation day 1 (Figure $5 \mathrm{a}$, lower panel). The levels of apoptotic cells were significantly higher than those in wild-type mice on lactation day 1 , but seemed to be closer to that in wild-type mice in mid-pregnancy (e.g. day 13 of pregnancy) (Figure $5 \mathrm{c}$ ). The rush of proliferation and differentiation during pregnancy-rescued ductal development in SirT1ko/ko mice manifested multiple features on lactation day 1 that could reassemble some characteristic features of wild-type mice in mid-pregnancy. Successive pregnancies, however, failed to improve the survival rate of either SirT1 $1^{+/ k o}$ pups or SirT1 ko/ko pups (three or four pregnancies in 3 months for each of three SirT1 ko/ko females tested), indicating that the lactation defect persisted. A SirT1 deficiency therefore affected ductal morphogenesis in virgin mice, and lobuloalveolar proliferation in parous mice.

\section{Deregulated negative feedback signals}

The characterization of lactation failure has revealed two developmental defects in SirT1 $1{ }^{\mathrm{ko}} / \mathrm{ko}$ female mice. The impeded ductal morphogenesis in virgin SirT1ko/ko mice bore some resemblance to the defective ductal morphogenesis seen in 
Figure 4

(a)
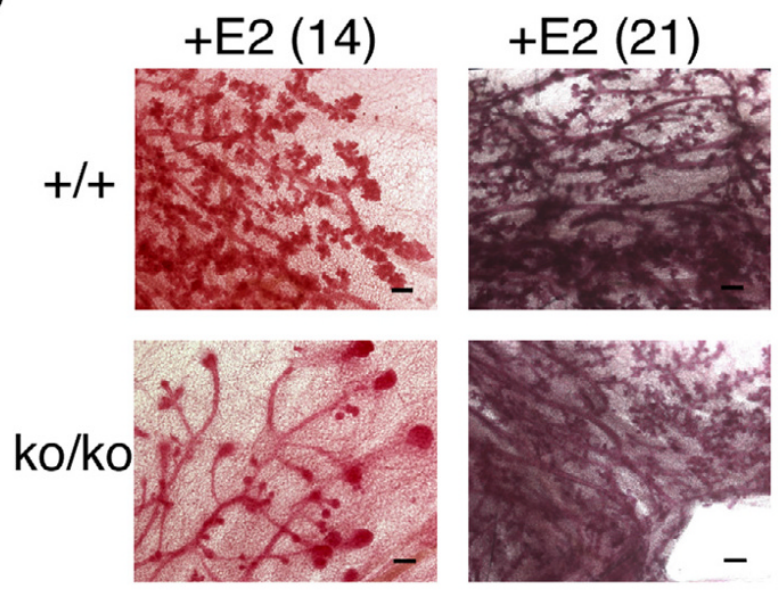

(b)

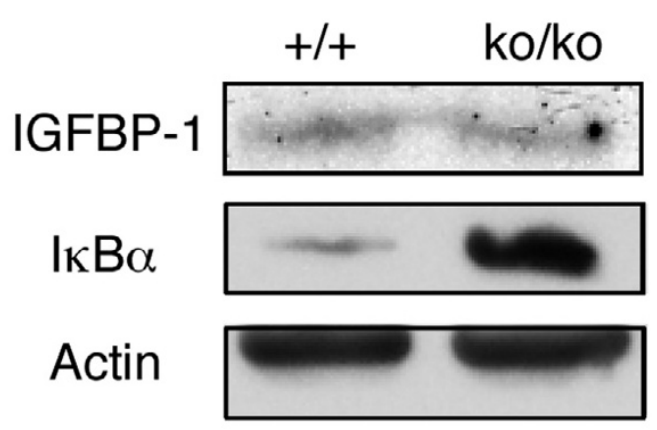

Estrogen implantation stimulates ductal elongation and site

branching. (a) Whole mount analysis of the mammary tissues from the virgin wild-type $(+/+)$ and SirT1 ko/ko mice implanted with estrogen pellets (+E2) on day 14 and day 21 . Scale bars $=200 \mu \mathrm{m}$. (b) Western blot analysis of the expression of insulin-like growth factor-1 binding protein-1 (IGFBP-1) and $\mathrm{I} \kappa \mathrm{B} \alpha$, with actin as a loading control, in mammary tissues from the virgin wild-type $(+/+)$ and $\mathrm{SirT} 1 \mathrm{ko} / \mathrm{ko}$ mice implanted with estrogen pellets on day 21 .

mice lacking $\mathrm{GH}$, estrogen, or IGF-1, as well as in mice harboring mutations in the corresponding receptors, such as the $\mathrm{GH}$ receptor and $E R \alpha[17,24-31]$. These previous studies have established the notion that $\mathrm{GH}$ and estrogen synergistically stimulate ductal morphogenesis, which is mediated by stromal cell-produced local IGF-1 in mammary tissues. Impeded ductal morphogenesis in virgin SirT1 ko/ko mice is therefore probably due to a deficit in IGF-1 signaling.

In addition to this early defect, pregnancy-induced mammary gland development in SirT1 ko/ko mice appeared to be arrested at the beginning of lobuloalveolar development, which is reminiscent of the phenotype in mice lacking $1 \kappa \mathrm{B}$ kinase alpha (IKK $\alpha$ ) activity for NF-KB activation [37]. IKK $\alpha$-deficient mice exhibit normal ductal morphogenesis at the onset of puberty and exhibit normal ductal side branching during early pregnancy. Owing to the absence of NF- $\mathrm{BB}$-dependent cyclin $\mathrm{D}_{1}$ expression, however, IKK $\alpha$-deficient mice display defective alveolar epithelial cell proliferation and fail to lactate [37]. While SirT1 negatively regulates the transcription activity of FoxOs and NF- $\mathrm{KB}$ in mammalian cells $[10,11,20]$, SirT1 ko/ko mice displayed the loss-of-function phenotypes. We hypothesized that SirT1 deficiency deregulates the expression of negative feedback signals and thereby desensitizes the cells to various types of stimulation. Indeed, among the many FoxOs and NF- $\kappa B$-regulated genes, IGFBP-1 and IKB $\alpha$ encode negative feedback signals for IGF-1 signaling and NF- $\kappa B$ activation, respectively. We found that the basal levels of both IGFBP-1 and I $\mathrm{KB} \alpha$ were increased in adipose tissues from virgin SirT1 ko/ko mice and in ductal epithelial cells from SirT1 ko/ko mice on lactation day 1 (Figure 3b). Meanwhile, the expression pattern of IGFBP-1 and IKB $\alpha$ in wild-type mammary tissues, shown in Figure $3 b$, was in agreement with the findings of others $[16,38,39]$. Namely, the expression of IGFBP-1 was downregulated during pregnancy, whereas NF- $\mathrm{BB}$ activity was increased at a late stage of pregnancy. The loss of SirT1 therefore increased the basal level of both IGFBP-1 and I $\mathrm{KB} \alpha$ in multilineages of cells, and potentially increased thresholds for activating SirT1-modulated signaling pathways.

SirT1 deficiency did not affect the ability of mammary epithelial progenitor cells to differentiate into functional alveolar epithelial cells despite the lactation failure (Figure 2c). SirT1 deficiency may therefore alter the homeostasis of the signals for ductal morphogenesis and/or the cellular response to the signals. Increased expression of both IGFBP-1 and IKB $\alpha$ in adipose tissues and mammary epithelial cells could simply reflect a global deregulation of gene expression in SirT1 ko/ko cells in which the activity of both FoxOs and NF- $\mathrm{KB}$ is increased. IGFBP-1 is a potent inhibitor of IGF-1 in vivo [40], suggesting that the elevated basal level of IGFBP-1 in adipose tissues in SirT1 ko/ko mice could reduce the effect of IGF-1. Moreover, deregulated IGFBP-1 expression, but not deregulated IKB $\alpha$ expression, was reversed in response to increased levels of estrogen in the mammary fat pads after implantation (Figure 4b). The finding is relevant to the fact that the expression of IGFBP-1 is downregulated as the level of estrogen increases in pregnant wild-type mice (Figure $3 \mathrm{~b}$ ). Increased levels of estrogen therefore stimulate the production of stromal cellderived local IGF-1, which overcomes the IGFBP-1 barrier in SirT1 ko/ko mice and, ultimately, reverses the increased levels of IGFBP-1 in mammary tissues.

It was noted that increased levels of estrogen and local IGF-1 did not interfere with the expression of $1 \kappa B \alpha$ (Figure $4 \mathrm{~b}$ ). To determine whether deregulated $I \kappa B \alpha$ expression in SirT1 ko/ko cells can be reversed, we treated MEFs with TNF $\alpha$ and measured the kinetics of $\mathrm{I} \kappa \mathrm{B} \alpha$ expression. Three independent lines of SirT1ko/ko MEFs were used, which displayed varying basal levels of increased $I \kappa B \alpha$ expression. Following $I \kappa B \alpha$ degradation induced by TNF $\alpha$, the levels of newly synthesized $1 \kappa B \alpha$ in all three SirT1 ko/ko MEFs were always higher than that of wild- 
(a)

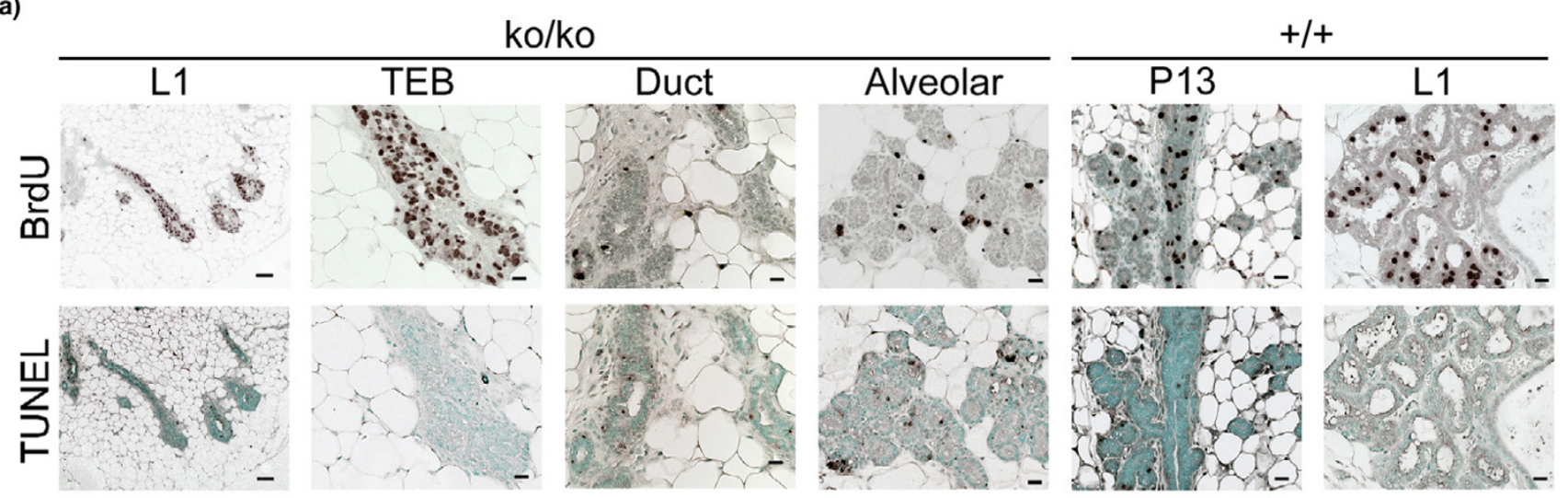

(b)

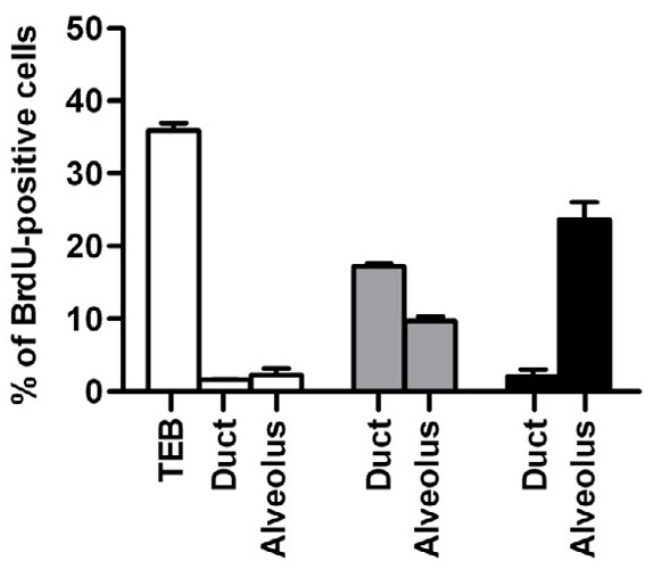

(c)

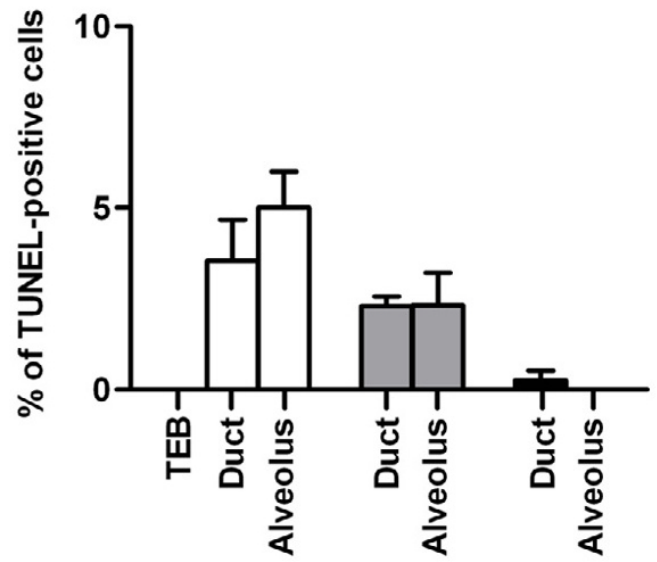

Mammary epithelial cell proliferation and apoptosis in SirT1ko/ko mice on lactation day 1. (a) Upper panel: bromodeoxyurdine (BrdU) staining of the mammary sections from SirT1 ko/ko $(\mathrm{ko} / \mathrm{ko})$ and wild-type $(+/+)$ mice on day 13 of pregnancy (P13) and lactation day 1 (L1). From left to right, three terminal end buds (TEBs) at a scale of $100 \mu \mathrm{m}$, a single TEB, ducts, and alveolus of SirT1 ko/ko mice at a scale of $20 \mu \mathrm{m}$, and ducts and alveolus of wild-type mice on P13 and L1 at a scale of $20 \mu \mathrm{m}$. Lower panel: TUNEL (Terminal deoxynucleotidyl transferase mediated dUTP Nick End Labeling) assay for apoptotic cells using sections neighbored to the sections for BrdU staining. (b) The quantitative analysis of BrdU-positive epithelial cells in TEBs, ducts, and alveoli of SirT1 ko/ko mice on L1 (open bar) and wild-type mice on P13 (grey bar) and L1 (solid bar). (c) The quantitative analysis of TUNEL-positive cells in neighboring sections.

type MEFs (Figure 6). This in vitro finding implies that SirT1 deficiency affects NF- $\kappa B$ signaling when the I $\mathrm{B}$ B kinase activation is normal.

Our current system might not be suitable for studying the effects of SirT1 deficiency on NF- $\kappa B$ activation in vivo. NF- $\kappa B$ signaling exhibits distinct biological responses because the expression of $\mathrm{I} \kappa \mathrm{B} \alpha$ depends on NF- $\kappa \mathrm{B}$ activation while the other isoforms of $I \kappa B(\beta$ and $\varepsilon$ ) are independent of NF- $\kappa B$ feedback [41]. For example, TNF $\alpha$ stimulation induces dampened oscillatory behavior after the first hour, whereas lipopolysacharide treatment leads to stable NF- $\kappa B$ activation $[42,43]$. The NF- $\mathrm{KB}$ signaling in the developing mammary gland is under the temporal control of RANK signaling and IKK $\alpha$ activation [37]. The finding of increased levels of newly synthesized $1 \kappa \mathrm{B} \alpha$ within the first hour indicated that SirT1 deficiency could dis- rupt the temporal control of NF- $\mathrm{KB}$ signaling to all types of stimulation (Figure 6). It remains unknown, however, whether the reduced IGF-1 signaling would affect the efficacy of RANK signaling and IKK $\alpha$ activation in developing mammary glands. The fact that individual SirT1 ko/ko mice manifested varying degrees of ductal morphogenesis (Figure 3a) makes it difficult to dissect the potential compound effect of SirT1 deficiency on both upstream IKK $\alpha$ activation and downstream NF- $\kappa B$ signaling. Other experimental systems, such as mammary epithelial cell-specific SirT1ko/ko female mice, may be used to address this issue.

\section{Discussion}

Our study of SirT1 ko/ko mice has unveiled a regulatory mechanism by which SirT1 modulates the efficacy of estrogen-stimulated local IGF-1 signaling for ductal morphogenesis. SirT1 
Figure 6

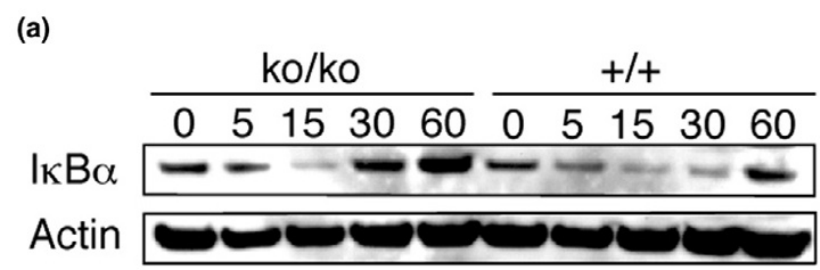

(b)

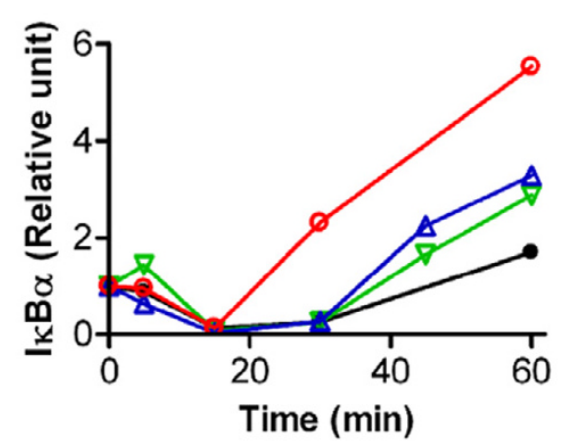

SirT1 deficiency derails NF- $\mathrm{KB}$ signaling in response to TNF $\alpha$ stimulation. (a) Western blot analysis of IkB $\alpha$ expression in SirT1 ko/ko (ko/ ko) and wild-type $(+/+)$ murine embryonic fibroblasts (MEFs) within the first hour after TNF $\alpha$ stimulation. Actin is used as a loading control. (b) The relative levels of newly synthesized IKB $\alpha$ in three independent SirT 1 ko/ko MEF lines (red circles, blue and green triangles) and wild-type MEFs after TNF $\alpha$ stimulation. For each line of MEFs, the unit of IKB $\alpha$ protein at each time point is relative to the unit 0 minutes after normalization with actin.

deficiency alters the homeostasis of gene expression and attenuates the efficacy of IGF-1 signaling. As a result, SirT1 ko/ ko mice manifest partial perinatal lethality and lactation failure phenotypes, implicating the role of evolutionarily conserved SirT1 in both postnatal survival and offspring survival of mammals.

\section{SirT1 balances the homeostasis of IGF-1 and IGFBP-1 in vivo}

SirT1 is an integral part of the IIS system that checks and balances the efficacy of insulin and IGF-1 signals. The IGF-1 signaling of the IIS system is required for survival after birth. As illustrated in Figure 7a, the function of SirT1 parallels the linear IGF-1/IGF-1 receptor/phosphoinositide-3'-OH kinase/Akt signaling pathway, both of which can negatively regulate the transcription activity of FoxOs. Mice harboring targeted mutations in either IGF-1 or the IGF-1 receptor gene exhibit perinatal lethality and growth retardation $[44,45]$. In the IGF-1 receptorexpressing cells, the binding of insulin and/or IGF-1 activates the phosphoinositide-3'-OH kinase and Akt kinase cascade for survival $[8,9,13]$. The phosphoinositide-3'-OH kinase/Akt signaling pathway is also highly conserved from worms to mammals, and functions to modulate energy metabolism and lifespan in lower organisms. The mammalian Akt kinase family
Figure 7 (a)

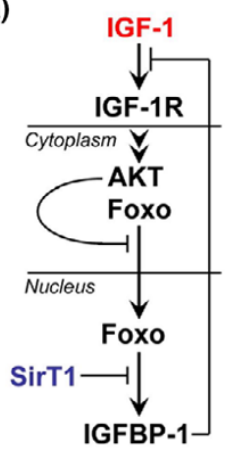

(b)

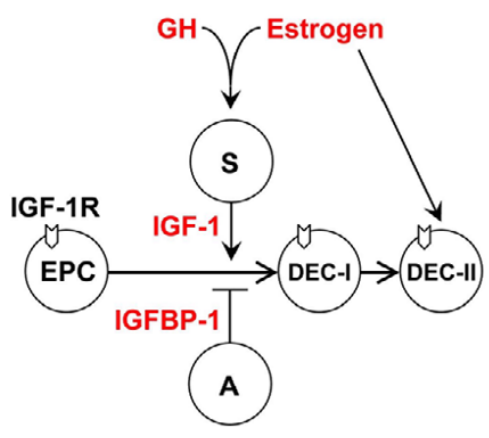

SirT1 modulates estrogen-insulin-like growth factor-1 signaling for ductal morphogenesis: a model. (a) The insulin/insulin-like growth factor-1 (IGF-1) signaling system: both SirT1 and Akt kinases can negatively regulate the transcription activity of forkhead box 'other' protein (FoxO) proteins. SirT1 deficiency deregulates the expression of insulin-like growth factor-1 binding protein-1 (IGFBP-1), which may exert autocrine and/or paracrine effects to inhibit IGF-1. (b) Mammary epithelial precursor cells (EPC) express IGF-1 receptor (IGF-1R) and can differentiate into estrogen receptor (ER)-negative ductal epithelial cells (DEC-I) in response to maternal, circulating, or estrogen-stimulated, stromal cell (S)-derived local IGF-1. At the onset of puberty, ovarian estrogen, in synergy with growth hormone $(\mathrm{GH})$, enhances the production of local IGF-1 and stimulates the differentiation of DEC-I to ER-positive ductal epithelial cells (DEC-II). SirT1 deficiency deregulates the expression of IGFBP-1 in adipose tissues (a), however, which attenuates the efficacy of the IGF-1 signaling and causes impeded ductal morphogenesis in virgin SirT1 ko/ko mice. Either pregnancy or exogenous estrogen can overcome the impeded ductal morphogenesis in virgin SirT 1 ko/ko mice and can stimulate the differentiation of EPC.

has three members, Akt1-Akt3, which provide specificity and versatility. Mice lacking both the Akt1 and Akt2 genes display perinatal lethal and growth retardation phenotypes that are strikingly similar to that of either IGF-1 or IGF-1 receptor-deficient mice [46]. In this regard, SirT1 ko/ko mice, as well as other strains of SirT1-deficienct mice, also exhibit perinatal lethal and growth retardation phenotypes (Figure 1c and Table 1) $[22,23]$. The consistent observation of perinatal lethal and growth retardation phenotypes from our study and studies with mice lacking IGF-1, the IGF-1 receptor, or Akt1/Akt2 suggests that upregulated FoxO activity unleashes the expression of downstream effector genes that lead to the birth-related stress. This birth-related stress may be related to the switch from a maternal level of IGF-1 to a neonatal level of IGF-1 because the surviving SirT1ko/ko mice can live into adulthood and can reproduce despite the growth retardation phenotype (Figure 1c). In this context, at least one of the FoxO-deficient mice strains, FoxO3 (Foxo3a/FKHRL1)-deficient mice, grow normally and develop functional mammary glands [47].

IGFBP-1 is one of six IGF-1 binding proteins, but is the only one that can be negatively regulated by insulin [48]. IGF-1 binding proteins inhibit the action of local IGF-1 in a spatial and temporal manner $[40,49]$. In mammalian cells, FoxO3 can directly bind to the promoter of the IGFBP-1 gene and activate 
the transcription [50-52]. SirT1 acts to negatively regulate the activity of nuclear $\mathrm{FoxO} 3$ and to decrease the expression of IGFBP-1 when the IGF-1 signal is low (Figure 7a). Increased levels of IGFBP-1 can diminish the action of IGF-1 signals in vivo. In that way, SirT1 balances the homeostasis of IGF-1 and IGFBP-1 for varying levels of IGF-1 signals.

The homeostasis of IGF-1 and IGFBP-1 clearly affects fertility, as shown by the infertility phenotype in both IGF-1-deficient mice and IGFBP-1 transgenic mice $[28,40,49]$. The serum level of estrogen in IGF-1-deficient female mice appears significantly reduced, whereas in ER $\alpha$-deficient mice, which are also infertile, there is a threefold increase [53]. In contrast, SirT1 ko/ko mice can be fertile despite the fact that the fertility was reduced as compared with that of wild-type females (Table 1). Although our preliminary assessment indicated that the serum level of estrogen in SirT1 ko/ko female mice was not overtly altered, it remains possible that a subtle irregularity or an individual variation of the estrogen level or ER $\alpha$ expression might be attributed to the reduced fertility. An alternative explanation for reduced fertility is that SirT1 deficiency deregulates the activity of FoxO3 and manifests an opposite effect of FoxO3 deficiency. FoxO3-deficient mice exhibit early depletion of ovarian follicles, which is reminiscent of premature ovarian failure in women [47,54]. Premature ovarian failure manifests as early cessation of ovarian function and as premature menopause caused by genetic mutations, chemo/radiation therapy, or other unknown factors. It is possible that SirT1 deficiency counterbalances normal release of ovarian follicles and reduces the fertility in mice.

\section{SirT1 modulates the efficacy of the IGF-1 signal for ductal morphogenesis}

We hypothesize that, in virgin SirT1 ko/ko mice, increased levels of IGFBP-1 in adipose tissues effectively diminish IGF-1 signals, including the estrogen-IGF-1 signaling, and halt the differentiation of mammary epithelial progenitor cells (Figure 7b). Several results of this study provided support for this hypothesis. First, the expression of IGFBP-1 is increased in mammary fat pads from virgin SirT1 ko/ko mice, in which there is no evidence of any duct (Figures 2a and 3a). Second, both pregnancy and estrogen implantation can rescue impeded ductal morphogenesis in SirT1 ko/ko mice (Figures 3a and 4a), demonstrating that increased levels of estrogen can enhance the production of local IGF-1. Moreover, the varying degrees of rescue among individual mice, as well as individual mammary fat pads of the same mouse, also indicated that local IGF1 must be halted in SirT1 ko/ko mice (Figure 3a, and data not shown). Finally, increased levels of estrogen specifically activate the IGF-1 signaling in mammary tissues, as shown by the reversal of IGFBP-1 expression in SirT1 ko/ko mice after estrogen implantation (Figure 4b). These results indicate that a gradient of IGF-1 signals, including both circulating and local IGF1 , may regulate mammary gland development at embryonic, postnatal, and reproductive stages, and that increased expression of IGFBP-1 decreases the efficacy of IGF-1 signals.

Mammary development may undergo estrogen/IGF-1-independent phases and estrogen/IGF-1-dependent phases, which encompasses a late stage of embryonic development to the onset of puberty and involves maternal IGF-1, circulating IGF-1, and estrogen-stimulated local IGF-1. By embryonic day 16.5, mammary mesenchymes are connected to primitive fat pads and begin to proliferate, which results in rudimentary ducts $[17,25,55,56]$. We propose that this process marks the transition from estrogen/IGF-1-independent mammary stem cells to IGF-1-dependent epithelial progenitor cells, and that maternal IGF-1 is sufficient to stimulate the differentiation of epithelial progenitor cells to estrogen-independent ductal epithelial cells but not estrogen-dependent epithelial cells (Figure $7 b$ ). This explains why the development of rudimentary ducts is normal in either IGF-1 or ER $\alpha$ knockout mice and that TEBs form and regress immediately before and after birth, respectively $[17,26,27]$. During the prepubertal period, the rudimentary ducts grow isometrically under the influence of circulating IGF-1. Like maternal IGF-1, circulating IGF-1 is not sufficient to induce the differentiation of estrogen-independent ductal epithelial cells into estrogen-dependent epithelial cells, which will express ER $\alpha$ and become estrogen dependent (Figure $7 b$ ). At the onset of puberty, ovarian estrogen, in synergy with pituitary $\mathrm{GH}$, causes a surge in the production of stromal cellderived local IGF-1 and stimulates the differentiation of estrogen-independent ductal epithelial cells to estrogen-dependent epithelial cells, which begins postnatal development of mammary gland (Figure 7b). Impeded ductal morphogenesis results in virgin SirT1 ko/ko mice because local IGF-1 is apparently not sufficient to overcome increased IGFBP-1 in mammary adipose tissues. SirT1 therefore positively regulates the efficacy of the estrogen-IGF-1 signaling for ductal epithelial cell proliferation and differentiation.

\section{The potential effect of modulating SirT1 activity}

Breast cancer results from the accumulation of inherited and/ or somatic mutations. Lifetime exposure to estrogen is a major risk factor for breast cancer, and a number of lifestyle factors, such as diet, body fat, alcohol consumption, exercise, parity, and hormone replacement therapy, may influence a woman's exposure to estrogen. The compound personal risk to a woman is difficult to assess because the molecular link between these factors and the effect of estrogen is poorly understood. The result of this study demonstrated that SirT1 positively modulates the efficacy of the estrogen-IGF-1 signal. This suggests that if SirT1 serves as a lifetime sensor to dietary restriction and acute withdrawal of nutrients [14,57], its activity could ultimately influence the risk of breast cancer. Interestingly, an epidemiological study of Dutch famine in 1945 indicated that an exposure to famine at prepubertal age increased the risk of breast cancer, most clearly seen in women who never gave birth [58]. Moreover, the serum levels 
of estrogen and IGF-1 were increased among those exposed to the famine, which suggests that an epigenetic adaptation phenomenon may take place. Assessing the potential correlation between the enzymatic activity of SirT1 and the risk of breast cancer is therefore of great interest in order to identify feasible targets for chemoprevention against breast cancer.

Several dietary polyphenols as well as small molecules have been identified as either agonists or antagonists of SirT1 $[59,60]$. The pharmacological effects of these compounds in humans remain elusive given that SirT1 targets multiple transcription factors and exerts pleiotropic effects. Nonetheless, our study of SirT1 ko/ko mice demonstrated the potential utility of SirT1 antagonists. Specifically, reducing SirT1 activity alters the homeostasis of cellular responses, including deregulation of the expression of IGFBP- 1 and $I \kappa B \alpha$, which can attenuate the stimulation from the estrogen-IGF-1 signaling and potentially desensitize mammary epithelial cells to NF- $\kappa B$ activation. SirT1 is therefore a candidate target for chemoprevention against breast cancer.

\section{Conclusion}

The study of mammary gland development is an excellent model system for unraveling how SirT1 modulates the efficacy of the estrogen-IGF-1 signaling and regulates the timing of ductal morphogenesis. These new mechanistic insights may also aid in understanding the role of SirT1 in breast cancer as well as in other organs in which local IGF-1 plays an important role.

\section{Competing interests}

The authors declare that they have no competing interests.

\section{Authors' contributions}

$\mathrm{HL}$ characterized the phenotypes of the SirT1 mutant mice, participated in the design of the study, and helped to draft the manuscript. GKR and NL helped to characterize the phenotypes. CW generated the SirT1 mutant embryonic stem cells and mice. BPR helped analyze the histology data. YG conceived of the study, participated in its design and coordination, and drafted the manuscript. All authors read and approved the final manuscript.

\section{Additional files}

The following Additional files are available online:

\section{Additional file 1}

A pdf file containing the materials and methods for the generation of SirT1 ko/ko mice. This supporting file documented the details of materials and methods used to generate both SirT1 co/co mice and SirT1 ko/ko mice. See http://www.biomedcentral.com/content/ supplementary/bcr1632-S1.pdf

\section{Acknowledgements}

The study is supported by grants from the American Cancer Society (RSG-04-019-01-CNE) and the Nathan Shock Center of Excellence in the Biology of Aging at the University of Washington (to YG). The authors thank Jennifer Blasi, Thomas Gernon, Janet Leath, Jennifer Lee, Bryce Sopher, Loraine Warner, and Heather-Marie Wilson for their contributions in the early phases of the study, and Dr Fred Alt, Dr Mary Helen Barcellos-Hoff, Dr Mark Groudine, Dr Warren Ladiges, Dr George Martin, Dr Peter Rabinovitch, and Dr Jeffrey Schwartz for their support and advice.

\section{References}

1. Imai S, Armstrong CM, Kaeberlein M, Guarente L: Transcriptional silencing and longevity protein Sir2 is an NAD-dependent histone deacetylase. Nature 2000, 403:795-800.

2. Landry J, Sutton A, Tafrov ST, Heller RC, Stebbins J, Pillus L, Sternglanz R: The silencing protein SIR2 and its homologs are NAD-dependent protein deacetylases. Proc Natl Acad Sci USA 2000, 97:5807-5811.

3. Aparicio OM, Billington BL, Gottschling DE: Modifiers of position effect are shared between telomeric and silent mating-type loci in S. cerevisiae. Cell 1991, 66:1279-1287.

4. Grunstein M: Molecular model for telomeric heterochromatin in yeast. Curr Opin Cell Biol 1997, 9:383-387.

5. Lin SJ, Defossez PA, Guarente L: Requirement of NAD and SIR2 for life-span extension by calorie restriction in Saccharomyces cerevisiae. Science 2000, 289:2126-2128.

6. Tissenbaum HA, Guarente L: Increased dosage of a sir-2 gene extends lifespan in Caenorhabditis elegans. Nature 2001, 410:227-230.

7. Fabrizio P, Gattazzo C, Battistella L, Wei M, Cheng C, McGrew K, Longo VD: Sir2 blocks extreme life-span extension. Cell 2005, 123:655-667.

8. Kenyon C: The plasticity of aging: insights from long-lived mutants. Cell 2005, 120:449-460.

9. Katic M, Kahn CR: The role of insulin and IGF-1 signaling in longevity. Cell Mol Life Sci 2005, 62:320-343.

10. Brunet A, Sweeney LB, Sturgill JF, Chua KF, Greer PL, Lin Y, Tran $\mathrm{H}$, Ross SE, Mostoslavsky R, Cohen HY, et al:: Stress-dependent regulation of FOXO transcription factors by the SIRT1 deacetylase. Science 2004, 303:2011-2015.

11. Motta MC, Divecha N, Lemieux M, Kamel C, Chen D, Gu W, Bultsma Y, McBurney M, Guarente L: Mammalian SIRT1 represses forkhead transcription factors. Cell 2004, 116:551-563.

12. Daitoku $\mathrm{H}$, Hatta M, Matsuzaki $\mathrm{H}$, Aratani $\mathrm{S}$, Ohshima T, Miyagishi $M$, Nakajima T, Fukamizu A: Silent information regulator 2 potentiates Foxo1-mediated transcription through its deacetylase activity. Proc Natl Acad Sci USA 2004, 101:10042-10047.

13. Datta SR, Brunet $A$, Greenberg ME: Cellular survival: a play in three Akts. Genes Dev 1999, 13:2905-2927.

14. Guarente L, Picard F: Calorie restriction - the SIR2 connection. Cell 2005, 120:473-482.

15. Baker ME: Steroid receptor phylogeny and vertebrate origins. Mol Cell Endocrinol 1997, 135:101-107.

16. Wood TL, Richert MM, Stull MA, Allar MA: The insulin-like growth factors (IGFs) and IGF binding proteins in postnatal development of murine mammary glands. J Mammary Gland Biol Neoplasia 2000 5:31-42.

17. Hovey RC, Trott JF, Vonderhaar BK: Establishing a framework for the functional mammary gland: from endocrinology to morphology. J Mammary Gland Biol Neoplasia 2002, 7:17-38.

18. Luo J, Nikolaev AY, Imai S, Chen D, Su F, Shiloh A, Guarente L, Gu W: Negative control of p 53 by Sir2alpha promotes cell survival under stress. Cell 2001, 107:137-148.

19. Vaziri H, Dessain SK, Ng Eaton E, Imai SI, Frye RA, Pandita TK, Guarente L, Weinberg RA: hSIR2(SIRT1) functions as an NADdependent p53 deacetylase. Cell 2001, 107:149-159.

20. Yeung F, Hoberg JE, Ramsey CS, Keller MD, Jones DR, Frye RA, Mayo MW: Modulation of NF- $\kappa \mathrm{B}-$ dependent transcription and cell survival by the SIRT1 deacetylase. EMBO J 2004, 23:2369-2380. 
21. Rodgers JT, Lerin C, Haas W, Gygi SP, Spiegelman BM, Puigserver P: Nutrient control of glucose homeostasis through a complex of PGC-1alpha and SIRT1. Nature 2005, 434:113-118.

22. McBurney MW, Yang X, Jardine K, Hixon M, Boekelheide K, Webb JR, Lansdorp PM, Lemieux M: The mammalian SIR2alpha protein has a role in embryogenesis and gametogenesis. Mo/ Cell Biol 2003, 23:38-54.

23. Cheng HL, Mostoslavsky R, Saito S, Manis JP, Gu Y, Patel P, Bronson R, Appella E, Alt FW, Chua KF: Developmental defects and p53 hyperacetylation in Sir2 homolog (SIRT1)-deficient mice. Proc Natl Acad Sci USA 2003, 100:10794-10799.

24. Silberstein GB: Postnatal mammary gland morphogenesis. Microsc Res Tech 2001, 52:155-162.

25. Parmar H, Cunha GR: Epithelial-stromal interactions in the mouse and human mammary gland in vivo. Endocr Relat Cancer 2004, 11:437-458.

26. Richards RG, Klotz DM, Walker MP, Diaugustine RP: Mammary gland branching morphogenesis is diminished in mice with a deficiency of insulin-like growth factor-I (IGF-I), but not in mice with a liver-specific deletion of IGF-I. Endocrinology 2004, 145:3106-3110

27. Mueller SO, Clark JA, Myers $\mathrm{PH}$, Korach KS: Mammary gland development in adult mice requires epithelial and stromal estrogen receptor alpha. Endocrinology 2002, 143:2357-2365.

28. Baker J, Hardy MP, Zhou J, Bondy C, Lupu F, Bellve AR, Efstratiadis A: Effects of an Igf1 gene null mutation on mouse reproduction. Mol Endocrinol 1996, 10:903-918.

29. Kleinberg DL: Early mammary development: growth hormone and IGF-1. J Mammary Gland Biol Neoplasia 1997, 2:49-57.

30. Walden PD, Ruan W, Feldman M, Kleinberg DL: Evidence that the mammary fat pad mediates the action of growth hormone in mammary gland development. Endocrinology 1998, 139:659-662.

31. Ruan W, Kleinberg DL: Insulin-like growth factor I is essential for terminal end bud formation and ductal morphogenesis during mammary development. Endocrinology 1999, 140:5075-5081.

32. Tsukamoto $\mathrm{Y}$, Kato J, Ikeda $\mathrm{H}$ : Silencing factors participate in DNA repair and recombination in Saccharomyces cerevisiae. Nature 1997, 388:900-903.

33. Mills KD, Sinclair DA, Guarente L: MEC1-dependent redistribution of the Sir3 silencing protein from telomeres to DNA double-strand breaks. Cell 1999, 97:609-620.

34. Mostoslavsky R, Chua KF, Lombard DB, Pang WW, Fischer MR, Gellon L, Liu P, Mostoslavsky G, Franco S, Murphy MM, et al.: Genomic instability and aging-like phenotype in the absence of mammalian SIRT6. Cell 2006, 124:315-329.

35. Gu Y, Seidl KJ, Rathbun GA, Zhu C, Manis JP, van der Stoep N, Davidson L, Cheng HL, Sekiguchi JM, Frank K, et al.: Growth retardation and leaky SCID phenotype of Ku70-deficient mice. Immunity 1997, 7:653-665.

36. Borghesani PR, Alt FW, Bottaro A, Davidson L, Aksoy S, Rathbun GA, Roberts TM, Swat W, Segal RA, Gu Y: Abnormal development of Purkinje cells and lymphocytes in Atm mutant mice. Proc Natl Acad Sci USA 2000, 97:3336-3341.

37. Cao Y, Bonizzi G, Seagroves TN, Greten FR, Johnson R, Schmidt $E V$, Karin M: IKK $\alpha$ provides an essential link between RANK signaling and cyclin $D_{1}$ expression during mammary gland development. Cell 2001, 107:763-775.

38. Clarkson RW, Heeley JL, Chapman R, Aillet F, Hay RT, Wyllie A, Watson CJ: NF- $\kappa B$ inhibits apoptosis in murine mammary epithelia. J Biol Chem 2000, 275:12737-12742.

39. Geymayer S, Doppler W: Activation of NF-kappaB p50/p65 is regulated in the developing mammary gland and inhibits STAT5-mediated beta-casein gene expression. FASEB $J$ 2000, 14:1159-1170.

40. Murphy $\mathrm{L}$ : Overexpression of insulin-like growth factor binding protein-1 in transgenic mice. Pediatr Nephrol 2000, 14:567-571.

41. Hoffmann A, Levchenko A, Scott ML, Baltimore D: The IkapppaBNF-kappaB signalling module:temporal control and selective gene activation. Science 2002, 298:1241-1245.

42. Covert MW, Leung TH, Gaston JE, Baltimore D: Achieving stability of lipopolysaccharide-induced NF-kappaB activation. Science 2005, 309:1854-1857.
43. Werner SL, Barken D, Hoffmann A: Stimulus specificity of gene expression programs determined by temporal control of IKK activity. Science 2005, 309:1857-1861.

44. Liu JP, Baker J, Perkins AS, Robertson EJ, Efstratiadis A: Mice carrying null mutations of the genes encoding insulin-like growth factor I (Igf-1) and type 1 IGF receptor (Igf1r). Cell 1993, 75:59-72.

45. Powell-Braxton L, Hollingshead P, Warburton C, Dowd M, PittsMeek S, Dalton D, Gillett N, Stewart TA: IGF-I is required for normal embryonic growth in mice. Genes Dev 1993, 7:2609-2617.

46. Peng XD, Xu PZ, Chen ML, Hahn-Windgassen A, Skeen J, Jacobs J, Sundararajan D, Chen WS, Crawford SE, Coleman KG, Hay N: Dwarfism, impaired skin development, skeletal muscle atrophy, delayed bone development, and impeded adipogenesis in mice lacking Akt1 and Akt2. Genes Dev 2003, 17:1352-1365.

47. Castrillon DH, Miao L, Kollipara R, Horner JW, DePinho RA: Suppression of ovarian follicle activation in mice by the transcription factor Foxo3a. Science 2003, 301:215-218.

48. Unterman TG, Glick RP, Waites GT, Bell SC: Production of insulin-like growth factor-binding proteins by human central nervous system tumors. Cancer Res 1991, 51:3030-3036.

49. Schneider MR, Lahm H, Wu M, Hoeflich A, Wolf E: Transgenic mouse models for studying the functions of insulin-like growth factor-binding proteins. FASEB J 2000, 14:629-640.

50. Guo S, Rena G, Cichy S, He X, Cohen P, Unterman T: Phosphorylation of serine 256 by protein kinase $B$ disrupts transactivation by FKHR and mediates effects of insulin on insulin-like growth factor-binding protein-1 promoter activity through a conserved insulin response sequence. J Biol Chem 1999, 274:17184-17192.

51. Tang ED, Nunez G, Barr FG, Guan KL: Negative regulation of the forkhead transcription factor FKHR by Akt. J Biol Chem 1999, 274:16741-16746.

52. Durham SK, Suwanichkul A, Scheimann AO, Yee D, Jackson JG Barr FG, Powell DR: FKHR binds the insulin response element in the insulin-like growth factor binding protein-1 promoter Endocrinology 1999, 140:3140-3146.

53. Hewitt SC, Korach KS: Progesterone action and responses in the alphaERKO mouse. Steroids 2000, 65:551-557.

54. Goswami D, Conway GS: Premature ovarian failure. Hum Reprod Update 2005, 11:391-410.

55. Hens JR, Wysolmerski JJ: Key stages of mammary gland development: molecular mechanisms involved in the formation of the embryonic mammary gland. Breast Cancer Res 2005, 7:220-224.

56. Mallepell S, Krust A, Chambon P, Brisken C: Paracrine signaling through the epithelial estrogen receptor alpha is required for proliferation and morphogenesis in the mammary gland. Proc Natl Acad Sci USA 2006, 103:2196-2201.

57. Nemoto $S$, Fergusson MM, Finkel $T$ : Nutrient availability regulates SIRT1 through a forkhead-dependent pathway. Science 2004, 306:2105-2108.

58. van Noord PA: Breast cancer and the brain: a neurodevelopmental hypothesis to explain the opposing effects of caloric deprivation during the Dutch famine of 1944-1945 on breast cancer and its risk factors. J Nutr 2004, 134:3399S-3406S.

59. de Boer VC, de Goffau MC, Arts IC, Hollman PC, Keijer J: SIRT1 stimulation by polyphenols is affected by their stability and metabolism. Mech Ageing Dev 2006, 127:618-627.

60. Heltweg B, Gatbonton T, Schuler AD, Posakony J, Li H, Goehle S, Kollipara R, Depinho RA, Gu Y, Simon JA, Bedalov A: Antitumor activity of a small-molecule inhibitor of human silent information regulator 2 enzymes. Cancer Res 2006, 66:4368-4377. 